\title{
Chronic erythropoietin treatment improves diet-induced glucose intolerance in rats
}

\author{
Corinne Caillaud', Mie Mechta ${ }^{2}$, Heidi Ainge', Andreas N Madsen ${ }^{2,3}$, Patricia Ruell', \\ Emilie Mas ${ }^{4}$, Catherine Bisbal ${ }^{5}$, Jacques Mercier ${ }^{5,6}$, Stephen Twigg ${ }^{7}$, Trevor A Mori ${ }^{4}$, \\ David Simar ${ }^{8}$ and Romain Barrès ${ }^{2}$
}

${ }^{1}$ Exercise Health and Performance, Faculty of Health Sciences, and Charles Perkins Centre, The University of Sydney, Sydney, New South Wales, Australia

${ }^{2}$ Faculty of Health and Medical Sciences, The Novo Nordisk Foundation Center for Basic Metabolic Research, University of Copenhagen, Copenhagen, Denmark

${ }^{3}$ Department of Neuroscience and Pharmacology, Faculty of Health Sciences, University of Copenhagen, Copenhagen, Denmark

${ }^{4}$ School of Medicine and Pharmacology, Royal Perth Hospital, The University of Western Australia, Perth, Western Australia, Australia

${ }^{5}$ UMR CNRS 9214, U1046 INSERM Physiologie et Médecine Expérimentale du Cœur et des Muscles, Université de Montpellier, Montpellier, France

${ }^{6}$ Physiology Department, CHU Arnaud de Villeneuve, Montpellier, France

${ }^{7}$ Department of Endocrinology, Sydney Medical School, Royal Prince Alfred Hospital, University of Sydney, Camperdown, New South Wales, Australia

${ }^{8}$ Inflammation and Infection Research, School of Medical Sciences, UNSW Australia, Sydney,

New South Wales, Australia

\author{
Correspondence \\ should be addressed \\ to C Caillaud or R Barrès \\ Emails \\ corinne.caillaud@sydney. \\ edu.au or barres@sund.ku.dk
}

\begin{abstract}
Erythropoietin (EPO) ameliorates glucose metabolism through mechanisms not fully understood. In this study, we investigated the effect of EPO on glucose metabolism and insulin signaling in skeletal muscle. A 2-week EPO treatment of rats fed with a high-fat diet (HFD) improved fasting glucose levels and glucose tolerance, without altering total body weight or retroperitoneal fat mass. Concomitantly, EPO partially rescued insulin-stimulated AKT activation, reduced markers of oxidative stress, and restored heat-shock protein 72 expression in soleus muscles from HFD-fed rats. Incubation of skeletal muscle cell cultures with EPO failed to induce AKT phosphorylation and had no effect on glucose uptake or glycogen synthesis. We found that the EPO receptor gene was expressed in myotubes, but was undetectable in soleus. Together, our results indicate that EPO treatment improves glucose tolerance but does not directly activate the phosphorylation of AKT in muscle cells. We propose that the reduced systemic inflammation or oxidative stress that we observed after treatment with EPO could contribute to the improvement of whole-body glucose metabolism.
\end{abstract}
Key Words
- glucose metabolism
- high-fat diet
- skeletal muscle
- myotubes

Journal of Endocrinology (2015) 225, 77-88

\section{Introduction}

Erythropoietin (EPO) is a glycoprotein synthesized by the kidney and plays a role in the regulation of red blood cell growth and survival. EPO, classically used for the treatment of anemia in chronic kidney disease, has recently emerged as a regulator involved in glucose metabolism. Results of early studies carried out of patients 
with end-stage renal disease (ESRD) indicated that EPO not only treated anemia but also improved insulin sensitivity (Borissova et al. 1993, Mak 1996). Results of other clinical studies of ESRD patients indicated that EPO treatment could improve fasting plasma insulin level (Tuzcu et al. 2004, Khedr et al. 2009) and control blood glucose (Spaia et al. 2000, Khedr et al. 2009). The results of these studies have indicated that improvements in glucose homeostasis and insulin sensitivity occurred before, or independently of increased hematocrit, indicating a metabolic role for EPO that is dissociated from hematopoiesis (Spaia et al. 2000). Animal models of long-term exposure to EPO showed clear positive effects on glucose metabolism. Transgenic mice constitutively overexpressing EPO displayed decreased body weight and improved glucose metabolism as compared with WT litter mates (Katz et al. 2010). Conversely, long-term exposure to EPO could prevent diet-induced obesity (Hojman et al. 2009). While a role of EPO in mediating muscle anabolism has been suggested, evidence that EPO directly targets skeletal muscle is still lacking.

Results of cell culture experiments indicated that EPO has the potential to directly target metabolic cells. Acute treatment with EPO protects pancreatic cells against the combined action of pro-apoptotic cytokines interleukin 1 (IL1 $\beta)$ tumor nexcosis factor alpha (TNF $\alpha$ ), and interferon gamma (IFN $\gamma$ ), suggesting indicative of its cytoprotective role (Maiese et al. 2005). In cultured adipocytes, EPO prevented dexamethasone-induced decrease in glucose uptake (Mikolás et al. 2012, Pan et al. 2013).

In this study, we investigated the effect of both acute and chronic EPO treatment on whole-body glucose metabolism and activation of the insulin signaling pathway in skeletal muscle. We found that $\mathrm{EPO}$ ameliorates diet-induced glucose intolerance and insulin signaling. We also show that EPO does not target the skeletal muscle cell indicating an indirect action through the improvement of several systemic factors known to be beneficial for whole-body glucose metabolism.

\section{Materials and methods}

\section{Chemicals, ELISA kits, and antibodies}

Palmitic acid endotoxin-free cell culture grade, anti-protease cocktail (P8340), and BSA (cell culture grade) were purchased from Sigma-Aldrich. Fetal bovine serum (FBS), minimum essential media alpha (MEM $\alpha$ ), and penicillin/streptomycin (P/S) antibiotic cocktail (15070-063) were from purchased Gibco. The experiments were conducted with human recombinant EPO (Janssen Cilag, Sydney, NSW, Australia). Primary antibodies were from Stressgen (heat-shock protein
72 (HSP72), SPA-810, 1/2000, Victoria, BC, Canada) or Cell Signaling (AKT (9272), P-AKT ${ }^{\text {(Ser473) }}$ (9271), Danvers, MA, USA), and secondary antibodies were purchased from Sigma-Aldrich (sheep anti-mouse, A5906) or Cell Signaling (goat anti-rabbit, 7074). Serum insulin and TNF $\alpha$ were measured using rat ultrasensitive ELISA Kits (Alpco, Laboratory Diagnostics (Caringbah, NSW, Australia) and R\&D Systems, Inc. (Minneapolis, MN, USA) respectively). Triglycerides (TGs) and cholesterol were measured using Thermo Scientific (Waltham, MA, USA) and Stanbio Kits respectively (Boerne, TX, USA). Blood glucose levels were measured using an AcuCheck II glucometer (Roche, Castle Hill, NSW). D-[U- $\left.{ }^{14} \mathrm{C}\right]$-glucose and scintillation liquid (Ultima gold) were obtained from Perkin Elmer (Waltham, MA, USA).

\section{Cell culture}

L6 and 3T3L1 cells were obtained from Lonza (Stockholm, Sweden). Human muscle primary cell cultures were established from muscle biopsies collected from male participants. The protocol was approved by the Local Ethics Committee (03/10/GESE, Montpellier, France) and informed and written consent was obtained from all participants. Myoblasts isolation, purification, and culture were performed as described previously (Barro et al. 2010). Muscle cells were grown in medium containing MEM $\alpha$ supplemented with $10 \% \mathrm{FBS}$ and $50 \mu \mathrm{g} / \mathrm{ml} \mathrm{P} / \mathrm{S}$ at $5 \% \mathrm{CO}_{2}$ and $37^{\circ} \mathrm{C}$. Once at confluence, cells were grown in MEM $\alpha, 2 \% \mathrm{FBS}$, and $50 \mu \mathrm{g} / \mathrm{ml} \mathrm{P} / \mathrm{S}$. All experiments were conducted at the myotube stage of differentiation. The medium was changed to $\mathrm{MEM} \alpha, 1 \% \mathrm{BSA}, \mathrm{P} / \mathrm{S}$, without serum $12 \mathrm{~h}$ before insulin stimulation. As a model of insulin-resistance, muscle cells were treated for $48 \mathrm{~h}$ with $500 \mu \mathrm{M}$ palmitate. The cells incubated with EPO $(10 \mathrm{U} / \mathrm{ml}$ final concentration) or palmitate were stimulated with 0,10 , and $100 \mathrm{nM}$ insulin for $5 \mathrm{~min}$. In the different experiments, cells were incubated with EPO for $5 \mathrm{~min}, 15 \mathrm{~min}$, or $48 \mathrm{~h}$. The cells were then washed twice in ice-cold phosphoprotein buffer $(20 \mathrm{mM}$ Tris, pH 7.4, $150 \mathrm{mM} \mathrm{NaCl}, 5 \mathrm{mM}$ EDTA, $150 \mathrm{mM} \mathrm{NaF}, 2 \mathrm{mM}$ $\mathrm{Na}_{3} \mathrm{VO}_{4}$, and $10 \mathrm{mM}$ pyrophosphate) and lysed (lysis buffer: $100 \mu \mathrm{l} 0.5 \mathrm{mM}$ phenylmethylsulfonyl fluoride (PMSF), protease inhibitor, and 1\% Triton X-100 in protein buffer). The cell lysates were centrifuged for $10 \mathrm{~min}$ at $4{ }^{\circ} \mathrm{C}$. The soluble fraction was collected and suspended in $100 \mu \mathrm{l}$ of a Laemmli buffer/ $\beta$-mercaptoethanol mixture and stored at $-20^{\circ} \mathrm{C}$.

\section{Glycogen synthesis}

Glycogen synthesis was measured using a modified version of a previously described method (Al-Khalili et al. 2003).

Published by Bioscientifica Ltd 
Briefly, L6 myotubes were incubated with EPO $(10 \mathrm{mU} / \mathrm{ml})$ for $24 \mathrm{~h}$, and then serum starved for $4 \mathrm{~h}$. After stimulation with insulin at 0 or $120 \mathrm{nM}$ for $30 \mathrm{~min}$, cells were supplemented with $\mathrm{D}-\left[\mathrm{U}-{ }^{14} \mathrm{C}\right]$-glucose for $90 \mathrm{~min}$ and lysed in $0.03 \%$ SDS. Carrier glycogen $(20 \mathrm{mg} / \mathrm{ml})$ was added and the glycogen pellet was precipitated using $98 \%$ ethanol. The samples were frozen at $-20^{\circ} \mathrm{C}$ overnight. The pellet was then resuspended in $300 \mu \mathrm{l}$ water and again incubated overnight at room temperature. Glucose ${ }^{14} \mathrm{C}$ was counted with a liquid scintillation counter (Hidex 300SL, Hidex, Turku, Finland).

\section{2-Deoxyglucose uptake}

Fully differentiated L6 myotubes were serum-starved in DMEM supplemented with $0.1 \% \mathrm{FBS}$ for $2 \mathrm{~h}$. The cells were then washed with Krebs-Ringer phosphate buffer $(10 \mathrm{mM}$ phosphate buffer, pH 7.4, $1 \mathrm{mM} \mathrm{MgSO}_{4}, 1 \mathrm{mM} \mathrm{CaCl}_{2}$, $136 \mathrm{mM} \mathrm{NaCl}$, and $4.7 \mathrm{mM} \mathrm{KCl}$ ) and incubated without or with insulin $(100 \mathrm{nM})$ for $20 \mathrm{~min}$ in the presence of Krebs-Ringer phosphate buffer supplemented with $0.1 \%$ BSA. Glucose transport was determined by the addition of 2 - $\left[{ }^{3} \mathrm{H}\right]$ deoxyglucose $(0.1 \mathrm{mM}$ and $0.5 \mu \mathrm{Ci} / \mathrm{ml})$. The reaction was stopped after $5 \mathrm{~min}$ by aspiration, and cells were washed four times with ice-cold PBS. The cells were lysed in Krebs-Ringer phosphate, 1\% Triton X-100, and glucose uptake was assessed by scintillation counting. Results were normalized for protein amount, which was measured by bicinchoninic acid (BCA) assay.

\section{Animal studies and experimental procedures}

The University of Sydney Ethics Committee (C42/ $1-2009 / 2 / 4921$ and C42/1-2009/3/4921) approved all animal experiments. All procedures complied with the Australian Code of Practice for the Care and Use of Animals for Scientific Purposes and the NSW Animal Research Act. Six-week-old male Sprague-Dawley rats were ordered from the Animal Resource Center (Canning Vale, WA, Australia). The animals were fed either a standard diet (16\% energy from fat, $59 \%$ from carbohydrates, $2.6 \mathrm{kcal} / \mathrm{kg}$, Rat and Mouse Breeder Food, Gordon's Specialty Stock Feeds (Yanderra, NSW, Australia)) or high-fat diet (HFD) (SF03-020, 45\% energy from fat, 40\% from carbohydrates, $4.78 \mathrm{kcal} / \mathrm{kg}$, Gordon's Specialty Stock Feeds). Acute AKT activation was measured in soleus muscle in a cohort of 16 adult (13-weeks-old) male rats fed standard diet. After 4-h fasting, animals received injections of high doses of EPO (i.p., $1300 \mathrm{U} / \mathrm{kg}$ ) in order to optimize potential activation of target pathways
(Christensen et al. 2012). The tissues were collected 60, 90, and 180 min following injection, snap-frozen in liquid nitrogen and stored at $-80^{\circ} \mathrm{C}$ until analysis was performed. Blood glucose levels were measured in a separate cohort of adult rats.

The effect of chronic EPO treatment in animals was investigated in 56 adult male rats. After a 1-week acclimatization to the animal house, the animals were fed either standard diet (CTL) or HFD for six and a half weeks. During the last 2 weeks, animals from each diet group were randomly assigned to receive i.p. injections of either vehicle (saline, CTL, and HFD groups) or EPO (500 U/kg EPO, three times a week, EPO or HFD/EPO groups). Food and caloric intake per $24 \mathrm{~h}$ was monitored throughout the duration of the study. After 2 weeks of EPO treatment, and $48 \mathrm{~h}$ after the sixth EPO injection, an intraperitoneal glucose tolerance test (IPGTT, $2 \mathrm{~g} / \mathrm{kg}$ glucose) was performed under fasting conditions and blood was collected from the tail vein for glucose and insulin analysis. The glucose-insulin index was calculated as previously reported (Marchionne et al. 2012). The animals were returned to their cage and received the final EPO injection. Two days later, after an overnight fast, animals were assigned to a nonstimulated (INS - ) or insulin-stimulated (INS + ) condition ( $1 \mathrm{U}$ insulin i.p./kg body weight). Soleus and extensor digitorum longus (EDL) muscles were collected $10 \mathrm{~min}$ after injection. In both acute and chronic EPO treatment studies, animals were killed by a lethal injection of Lethabarb $(325 \mathrm{mg} / \mathrm{ml}$ pentobarbitone sodium) followed by surgical removal of the heart.

\section{Western blotting analysis}

The cell lysates and tissue samples were analyzed by western blotting as described previously (Barrès et al. 2009). Briefly, the tissues were homogenized in a 10:1 (volume-to-weight) ratio of ice-cold extraction buffer (10 mM Tris-HCl (pH 7.4); $100 \mathrm{mM} \mathrm{NaCl} ; 1 \mathrm{mM}$ each of EDTA, EGTA, NaF, and PMSF; $2 \mathrm{mM} \mathrm{Na}_{3} \mathrm{VO}_{4} ; 20 \mathrm{mM}$ $\mathrm{Na}_{4} \mathrm{P}_{2} \mathrm{O}_{7} ; 1 \%$ Triton X-100; $10 \%$ glycerol; $0.1 \%$ SDS; 0.5\% deoxycholate; and $10 \mu \mathrm{g} / \mathrm{ml}$ protease inhibitor cocktail). Solubilized proteins were suspended in Laemmli buffer, heated for $5 \mathrm{~min}$ at $95^{\circ} \mathrm{C}$ or $20 \mathrm{~min}$ at $65^{\circ} \mathrm{C}$ (for phosphorylated proteins). Proteins $(20-90 \mu \mathrm{g})$ were separated by SDS-PAGE and transferred onto nitrocellulose membranes. The membranes were blocked, washed, and incubated overnight at $4{ }^{\circ} \mathrm{C}$ with primary antibody (P-AKT and total AKT 1/2000 in TBST 5\% BSA; HSP72, 1/2000 1\% milk). Following incubation with HRP-conjugated secondary antibodies, proteins were detected by ECL (Millipore,

Published by Bioscientifica Ltd 
Bayswater, VIC, Australia) and visualized using the ChemiDoc XRS + and Image Lab Software (Bio-Rad).

\section{RNA extraction and RT-PCR}

RNA extraction and RT-PCR was performed using a modified version of a previously described method (Barrès et al. 2012). In brief, RNA from rat bone marrow, rat skeletal muscle, and L6 cells was purified using TRIzol reagent according to the recommendations of the manufacturer. cDNA synthesis was performed using the iScript cDNA Synthesis Kit (Bio-Rad). PCR was performed in a final volume of $10 \mu \mathrm{l}$, consisting of diluted cDNA, SYBR Green PCR Master Mix, EPO receptor (EPOR) primer, and nuclease-free water. The samples were analyzed in triplicates. The primers were designed using Primer3 as follows: sense, AGGTGGACGTGTCAGCAGGC and antisense, CCCCGCAGGTTGCTCAGGAC.

\section{Oxidative stress}

F2-isoprostanes (F2-IsoPs) and isofurans (IsoFs), formed in vivo by free radical nonenzymatic oxidation of arachidonic acid (C20:4n-6), were quantified (Morrow et al. 1990, Henriksen et al. 2011). F2-IsoPs and IsoFs in soleus muscle were measured by gas chromatography-mass spectrometry using electron-capture negative ionization as previously described (Mas et al. 2011).

\section{Statistical analyses}

The results are presented as mean \pm s.D. All data were tested for normality and equality of variance. Data derived from western blots in the acute EPO studies were analyzed using a one-way ANOVA. The HFD and chronic EPO animal studies were analyzed with a two-way ANOVA (diet $\times$ EPO) or a one-way ANOVA when only three groups were analyzed (CTL, HFD, and HFD/EPO). Insulin stimulation in vivo was analyzed with a two-way ANOVA (ins $\times$ groups). Following ANOVA, post hoc analysis was performed using the Bonferroni's test for multiple comparisons. For cell culture experiments, a two-way ANOVA (insulin $\times$ EPO) was used for analysis of AKT activation following incubation with insulin and EPO once data were normalized via appropriate transformation. Nonparametric (Kruskal-Wallis or Wilcoxon-Mann-Whitney $U$ ) tests were used for palmitate, glycogen synthesis, and glucose uptake experiments. The correlations were investigated using the Pearson's product-moment coefficient. The significance level for all comparisons was set at $P<0.05$. The statistical analysis was conducted with SPSS (IBM).

\section{Results}

\section{Acute and chronic EPO injection in lean animals is associated with an increased phosphorylation of AKT in muscle}

We tested the effect of EPO on the activation of glucose metabolism in skeletal muscle by measuring the phosphorylation of AKT, a signaling node in the metabolic action of insulin. After an acute i.p. injection of EPO, AKT phosphorylation at serine 473 was elevated in soleus muscle with peak activation at 90 min (T0 vs 90 min: $t=-2.806, P<0.05$; Fig. $1 \mathrm{~A}$ and B). Despite enhanced phosphorylation of AKT in skeletal muscle, acute EPO did not affect glycemia within a $90 \mathrm{~min}$ period following injection (Fig. 1C), indicating that EPO-induced AKT signaling activation in vivo in skeletal muscle is not sufficient to alter whole-body glucose homeostasis. Treatment of lean animals with EPO for 2 weeks also resulted in increased basal AKT phosphorylation in soleus $(t=-2.21$, $P=0.045$; Fig. $1 \mathrm{D}$ and E).
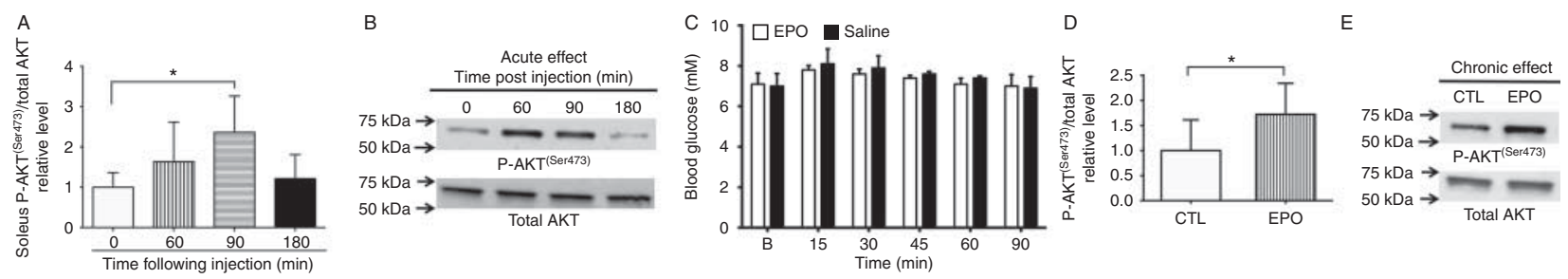

Figure 1

EPO activates AKT phosphorylation in rat soleus muscle. (A) Quantification of P-AKT/total AKT in soleus muscle following acute i.p. injection of $1300 \mathrm{IU}$ $\mathrm{EPO} / \mathrm{kg}(n=4)$ and (B) western blot images. (C) Blood glucose time course following i.p. injection of $1300 \mathrm{IU}$ EPO/kg $(n=4)$. (D) Quantification of
$\mathrm{P}-\mathrm{AKT} /$ total AKT following chronic EPO treatment ( $500 \mathrm{U} / \mathrm{kg}$, three times a week for 2 weeks) in chow-fed animals $(n=8)$ and (E) western blot images. $\star P<0.05$ indicates a statistically significant difference between groups. (c) 2015 The authors Printed in Great Britain
Published by Bioscientifica Ltd 
Table 1 Parameters assessed in this study

Animal characteristics at the start of
EPO treatment
Body weight week $4(\mathrm{~g})$
FBG $(\mathrm{mM})$
Animal characteristics at the end
of the study
Body weight $(\mathrm{g})$
TNF $\alpha(\mathrm{pg} / \mathrm{ml})$
Hct $(\%)$
TGs $(\mathrm{mM})$
Total cholesterol $(\mathrm{mg} / \mathrm{dl})$

\begin{tabular}{c} 
CTL \\
\hline$n=16$ \\
$336 \pm 24$ \\
$5.59 \pm 0.65$ \\
$n=8$ \\
$367 \pm 14$ \\
$0.70 \pm 0.67^{\ddagger}$ \\
$49.2 \pm 1.2$ \\
$0.29 \pm 0.11^{\ddagger}$ \\
$69.0 \pm 13.7$
\end{tabular}

\begin{tabular}{c}
\hline EPO \\
\hline \\
\\
\\
\\
\\
\\
$377 \pm 8$ \\
$0.77 \pm 0.67^{\ddagger}$ \\
$67.9 \pm 2.5^{\dagger}$ \\
$0.29 \pm 0.08^{\ddagger}$ \\
$48.4 \pm 17.2^{\ddagger}$ \\
\hline
\end{tabular}

\begin{tabular}{c}
\hline HFD \\
\hline$n=16$ \\
$392 \pm 35^{*}$ \\
$6.48 \pm 0.47^{*}$ \\
$n=8$ \\
$464 \pm 34^{\dagger}$ \\
$2.03 \pm 0.53^{\dagger}$ \\
$47.3 \pm 1.2$ \\
$0.43 \pm 0.20^{\dagger}$ \\
$75.6 \pm 11.2$ \\
\hline
\end{tabular}

\begin{tabular}{c}
\hline HFD/EPO \\
\hline \\
\\
\\
$n=8$ \\
$466 \pm 49^{\dagger}$ \\
$1.06 \pm 0.67^{\ddagger}$ \\
$61.9 \pm 1.9^{+, \neq}$ \\
$0.43 \pm 0.16^{\dagger}$ \\
$59.6 \pm 15.1^{\ddagger}$ \\
\hline
\end{tabular}

FBG, fasting blood glucose; Hct, hematocrit; TGs, triglycerides; TNF $\alpha$, tumor necrosis factor alpha; CTL, control, chow-fed treated with saline; HFD, high-fat diet treated with saline; EPO, chow-fed treated with erythropoietin; HFD/EPO, HFD treated with EPO. Statistical analysis was conducted using a two-way ANOVA (Diet $\times$ EPO). A Bonferroni correction was used for multiple comparisons and specific effects between groups. Animal characteristics at the start of EPO treatment: ${ }^{*} P<0.05, C T L$ versus HFD. Animal characteristics at the end of the study: $P<0.05$, ${ }^{\dagger}$ versus $C T L$ and ${ }^{\ddagger}$ versus HFD.

\section{EPO improves glucose tolerance and partially restores insulin-stimulated AKT phosphorylation in high-fat-fed rats}

The activation of AKT signaling following injection of EPO prompted us to determine the effect of repeated EPO treatment on glucose metabolism. Lean and diet-inducedobese rats were chronically treated with EPO for 2 weeks. We controlled for the fact that, at initiation of the EPO treatment, animals fed HFD were bigger than chow-fed animals and exhibited signs of impaired glucose metabolism, as shown by higher blood glucose $(P<0.05$; Table 1). At the completion of the study, the 6-week HFD resulted in increased fasting insulin and glucose levels ( $P<0.05$; Fig. $2 \mathrm{~A}$ and B). EPO lowered fasting blood glucose in HFD/EPO animals (diet $\times \mathrm{EPO}$ interaction: $F(1,32)=6.1, P<0.05$; Fig. $2 \mathrm{~A})$ compared with HFD, while serum insulin levels were not significantly affected (diet $\times \mathrm{EPO}$ interaction: $F(1,28)=2.1, P=0.15$; Fig. $2 \mathrm{~B}$ ). Animals fed with a HFD and treated with EPO also showed improved glycemic control as indicated by reduced blood glucose levels during an IPGTT $(F(1,28)=3.09, P=0.040$; Fig. 2C) with a significant difference between HFD and $\mathrm{HFD} / \mathrm{EPO}$ at $15 \mathrm{~min}$. Glucose area under the curve (AUC; Fig. 2D) was increased in the HFD group and normalized in the HFD/EPO group, while insulin AUC increased with HFD and was unchanged by EPO treatment (Fig. 2E). Accordingly, the glucose-insulin index was increased in the HFD group and reduced in the HFD/EPO group $(P<0.05$; Fig. 2F).

Energy intake was similar during the course of the study (Fig. 3A). Chronic EPO injection did not modify weight gain or fat accumulation in EPO or HFD/EPO rats compared with CTL and HFD respectively, as measured by body or retroperitoneal fat pad weights (Fig. 3B and C). These results indicate that EPO ameliorates glucose metabolism independently from changes in body weight or retroperitoneal fat mass. While circulating TGs were unchanged, total-cholesterol levels were decreased after EPO treatment (Table 1).

To determine if EPO treatment ameliorated insulinsensitivity in skeletal muscle, we injected insulin into fasting CTL, HFD, and HFD/EPO animals and assessed AKT phosphorylation in oxidative and glycolytic muscles. In soleus, there was an interaction between insulin and treatment groups $(F(1,46)=3.60, P=0.045$; Fig. $4 \mathrm{~A}$ and $\mathrm{B})$. Insulin markedly stimulated AKT phosphorylation in CTL $(t=-4.76, P<0.01)$, while HFD inhibited its action (Fig. 4A and B). Although EPO treatment restored insulinstimulated AKT phosphorylation in HFD/EPO compared with CTL, we did not find a significant difference compared with baseline level (Ins - versus Ins+, $P>0.05$ ). Increased baseline levels of P-AKT in this group could explain this result (Fig. 4A). In EDL, insulin-induced AKT phosphorylation was inhibited in HFD, yet EPO did not restore insulin signaling $(P>0.05$; Fig. $4 \mathrm{C}$ and $\mathrm{D})$.

\section{EPO treatment is associated with reduced inflammation, and oxidative stress, and increases HSP72 expression}

To determine the potential contributors of EPO-induced amelioration of glucose metabolism or AKT phosphorylation in muscle, we investigated the effect of chronic EPO on inflammation levels, oxidative stress, and expression of HSP72, a chaperone protein known to protect insulin signaling (Chung et al. 2008). Systemic levels of the

Published by Bioscientifica Ltd 
A
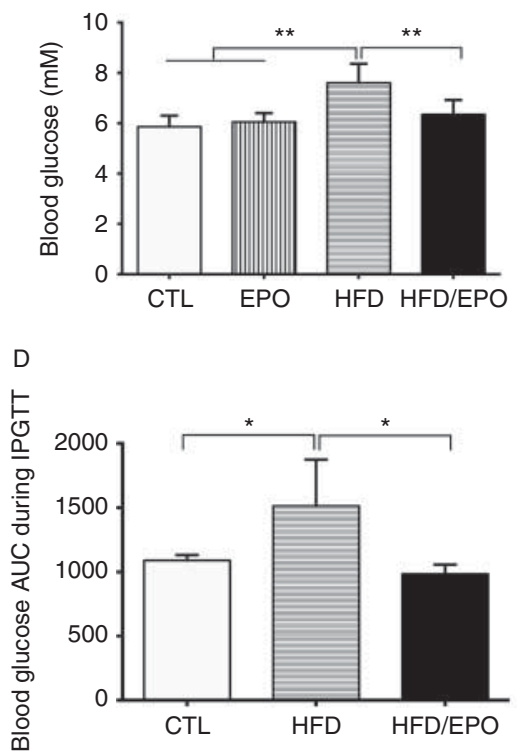

B

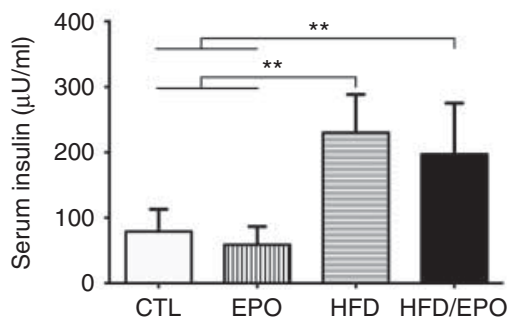

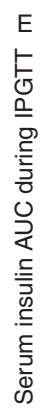

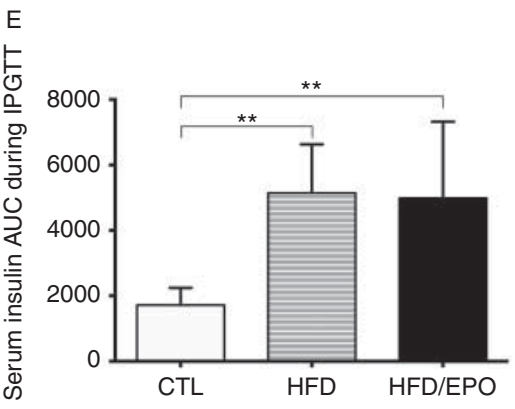

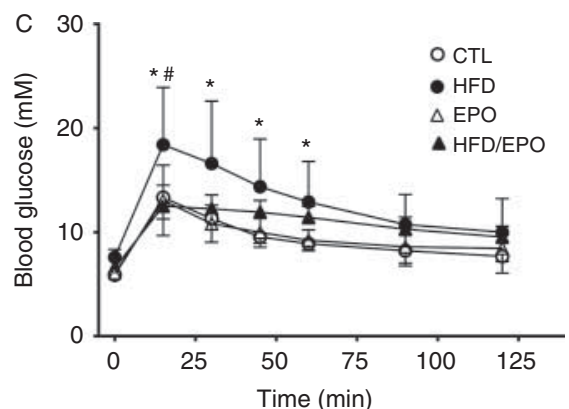

$\mathrm{F}$

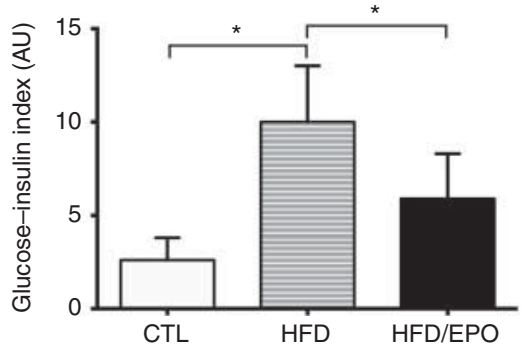

Figure 2

Short-term EPO treatment improves glucose metabolism. (A) Blood glucose levels and (B) serum insulin concentrations in treatment groups. (C) Blood glucose during an intraperitoneal glucose tolerance test (IPGTT). (D) Blood glucose area under the curve (AUC). (E) Serum insulin AUC and (F) glucoseinsulin index during IPGTT. $* P<0.05$ indicates a statistically significant

inflammatory cytokine TNF $\alpha$ were increased with HFD and reduced with $\mathrm{EPO}$ treatment (diet $\times \mathrm{EPO}$ interaction: $F(1,26)=5.54, P=0.026$, with a significantly lower levels in HFD/EPO compared with HFD; Table 1), indicating that EPO may participate in the decrease in systemic inflammation. To determine the role of EPO in oxidative stress in our model of HFD-induced glucose intolerance, we quantified both F2-IsoPs and IsoFs levels in muscle. Measurement of F2-IsoPs or IsoFs is a state-of-the-art approach for assessing oxidative stress in vivo in diverse disorders including obesity (Montuschi et al. 2004). EPO treatment decreased oxidative stress in soleus muscle as indicated by decreased IsoFs levels in the HFD/EPO group compared with HFD $(P<0.05$; Fig. 5A). The same trend was observed for F2-IsoP without reaching significance (Fig. $5 \mathrm{~B}$ ). We also showed that EPO treatment rescued the HFD-induced reduction in HSP72 expression in soleus $(F(1,26)=4.98, P=0.035$, HFD lower than all other groups, $P<0.05$; Fig. $5 \mathrm{C}$ and D).

\section{EPO does not directly target AKT phosphorylation in muscle cells}

We aimed to determine if EPOR was expressed in muscle cells and whether it could mediate metabolic difference between CTL and HFD shown in C or between groups as shown in figures for other panels. ${ }^{\#} P<0.05$ between HFD and HFD/EPO in $C$ and $* * P<0.01$ indicates a statistically significant difference between groups. CTL, control and saline; EPO, control and EPO; HFD, high-fat diet and saline; HFD/EPO, HFD and EPO; AU, arbitrary units; $n=8$ in each group.

action in vivo and in vitro. While we could not detect EPOR in soleus or EDL muscles, we found that EPOR was expressed at substantial levels in L6 myoblasts and myotubes (Fig. 6A). We then demonstrated that EPO had no significant effect on glucose uptake or glycogen synthesis in L6 myotubes incubated with EPO alone or co-incubated with EPO and insulin ( $P>0.05$; Fig. $6 \mathrm{~B}$ and $\mathrm{C}$ ). Given the previous results indicating that EPO activates the PI3K/AKT pathway in metabolic cells (Mikolás et al. 2012), we investigated the effect of EPO on AKT phosphorylation in differentiated rat muscle cell lines and human primary cells. We then first confirmed the existence of a synergy between EPO and insulin towards AKT phosphorylation using 3T3-L1 adipocytes treated with insulin and EPO $(P<0.05$; Fig. $7 \mathrm{~A}$ and $\mathrm{B})$. We then showed that EPO alone did not activate AKT in L6 myotubes acutely or chronically incubated with $10 \mathrm{U} / \mathrm{ml}$ EPO. When cells were stimulated with submaximal or maximal doses of insulin, EPO did not affect insulinstimulated AKT phosphorylation (Fig. 7C and D). However, EPO did improve AKT phosphorylation in rat myotubes exposed to palmitate (Fig. 7E and F). We also showed that, in human primary myotubes, EPO did not activate AKT and did not affect the action of insulin on

Published by Bioscientifica Ltd 

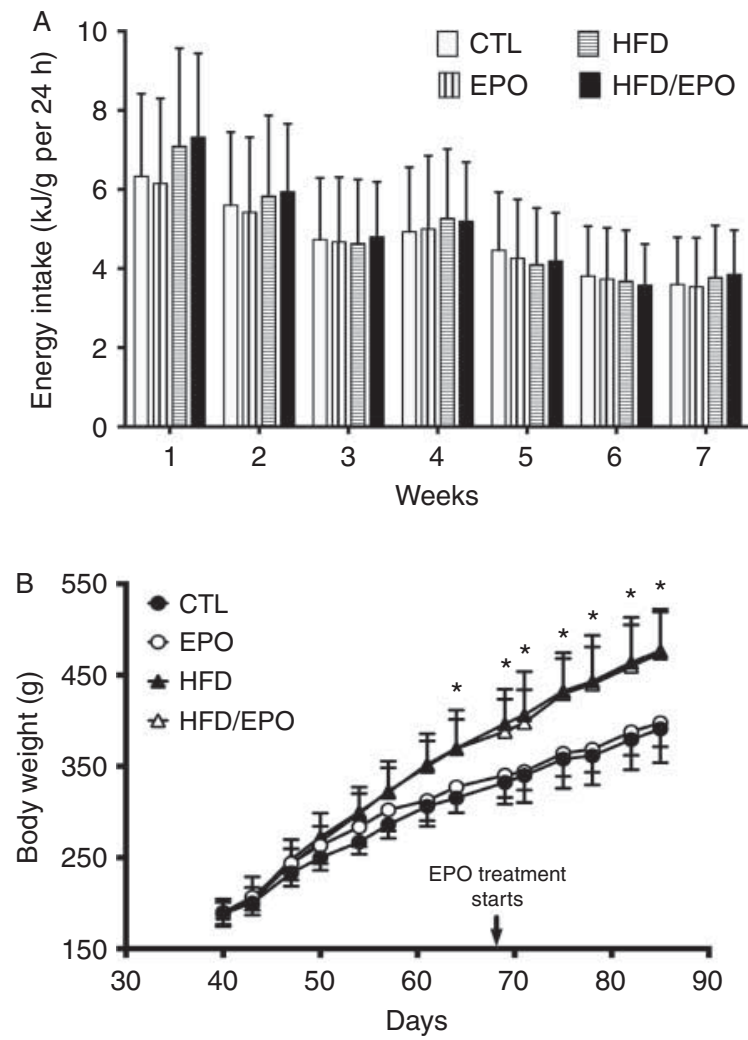

C

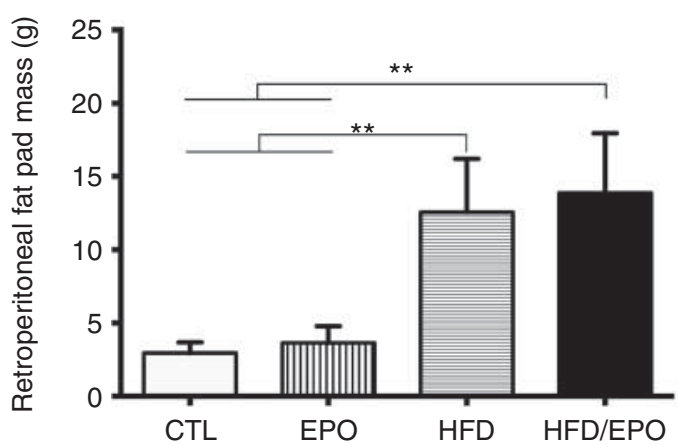

Figure 3

Short-term EPO treatment does not alter body weight gain or fat tissue mass. (A) Energy intake and (B) body weight during the course of the study. (C) Retroperitoneal fat pad mass at the end of the study. Statistical significance: $* P<0.05$ between chow- or HFD-fed animals and $* * P<0.01$ between groups. CTL, chow and saline; EPO, chow and EPO; HFD, high fat diet and saline; HFD/EPO, HFD and EPO; $n=8$ in each group.

P-AKT levels (Fig. 7G and H). Taken together, these results provide evidence that while EPOR is expressed in rat myotubes, EPO does not directly potentiate insulin action in muscle cells in vitro. However, EPO can rescue palmitate-induced inhibition of AKT activation, indicating that specific pathway activated by palmitate might have played a role. As EPOR was not detectable in soleus, our results also indicate that changes observed in vivo in soleus from EPOtreated animals resulted from an indirect action of EPO on this tissue.
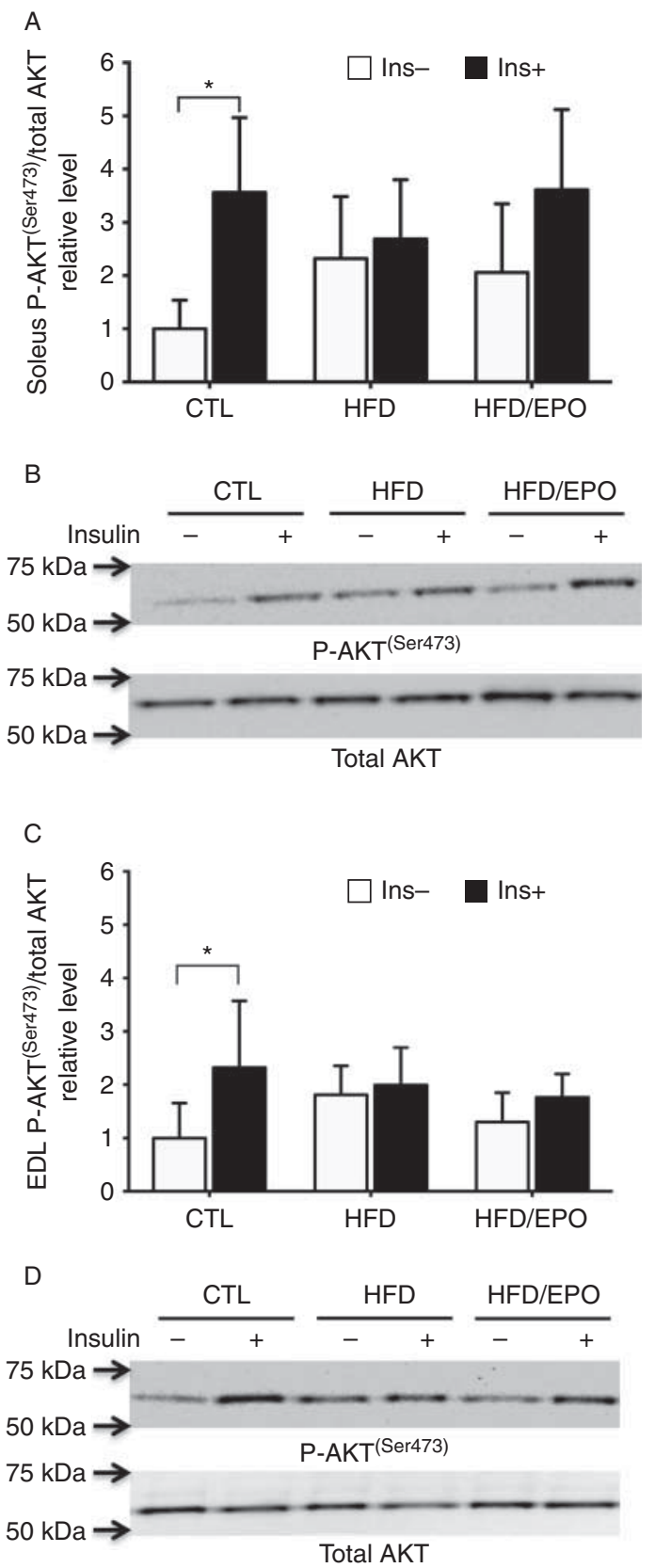

Figure 4

Chronic EPO treatment partially restores insulin-stimulated AKT phosphorylation in soleus muscle. Quantification and western blotting images showing P-AKT/total AKT in nonstimulated (Ins-) or in insulin-stimulated (Ins + ) conditions in ( $A$ and $B$ ) soleus and ( $C$ and D) EDL muscles. P-AKT/total AKT is expressed as fold of the value for the control nonstimulated condition. ${ }^{*} P<0.05$ indicates a statistically significant difference between conditions. CTL, control and saline; EPO, control and EPO; HFD, high-fat diet and saline; HFD/EPO, HFD and EPO; $n=8$ in each group.

Published by Bioscientifica Ltd 
A

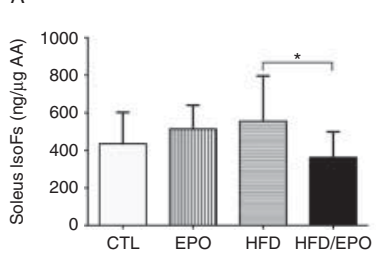

B

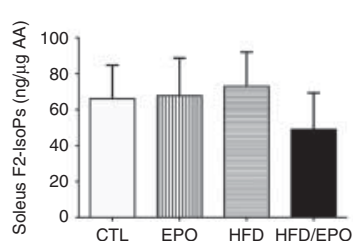

C

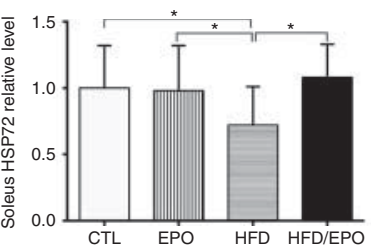

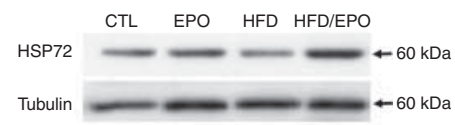

Figure 5

Chronic EPO reduces oxidative stress and restores HSP72 levels in soleus muscle. (A) Oxidative stress assessed by measurement of IsoFs and

(B) F2-IsoP levels in soleus muscle. (C) Quantification of HSP72 protein level.

(D) Representative western blot image. ${ }^{*} P<0.05$ indicates a statistically

\section{Discussion}

We report that EPO treatment improves glucose tolerance in diet-induced-glucose-intolerant rats. We further show that EPO modulates AKT phosphorylation in soleus, and provide evidence that EPO does not directly target the muscle cell to trigger this effect. We observed that EPO treatment is associated with a reduced systemic inflammation, lowered muscle oxidative stress, and increased muscle HSP72 expression, indicating that the improvement in glucose metabolism induced by EPO results from the amelioration of systemic factors.

Both acute and chronic EPO treatment in vivo led to AKT activation in soleus muscle from lean animals. A single injection of a high dose of EPO induced transient phosphorylation of AKT without altering systemic levels of glucose, indicating that EPO-induced phosphorylation of AKT in skeletal muscle has a limited role in acute glucose control in animals with normal blood glucose levels. In contrast, results described in a previous report indicated that acute EPO injection in streptozotocin-induced diabetic rats with elevated blood glucose levels (approximately $22 \mathrm{mM}$ ) significant difference between groups. CTL, control and saline; EPO, control and EPO; HFD, high-fat diet and saline; HFD/EPO, HFD and EPO; $n=8$ in each group.

led to a small (6\%) but significant decrease in blood glucose levels; however, the tissues involved have not been identified (Mikolás et al. 2012). Initial blood glucose levels may thus be critical in the acute effect of EPO, consistent with the study reporting that hyperglycemia is necessary for stimulation of EPO-induced glucose uptake in adipocytes (Mikolás et al. 2012). As we have also shown that rat soleus muscle did not express EPOR, AKT activation in our model is likely to be indirect via a still unknown mechanism (Wang et al. 2014). In human, acute i.v. injection of EPO was without effect on phosphorylation of AKT, AMP-activated protein kinase, or acetyl Co-A carboxylase, while insulin sensitivity and blood glucose levels were not affected (Christensen et al. 2012). The absence of AKT activation following acute EPO injection in that study may be due to the use of a much lower dose of EPO (200 and $400 \mathrm{IU} / \mathrm{kg}$ versus $1300 \mathrm{IU} / \mathrm{kg}$ in the current study), to the timing of the collection of muscle biopsies, to the fiber type composition of the muscle analyzed, or species-specific differences.

In our study, high-fat-fed animals presented increased body weight and retroperitoneal fat mass, elevated fasting

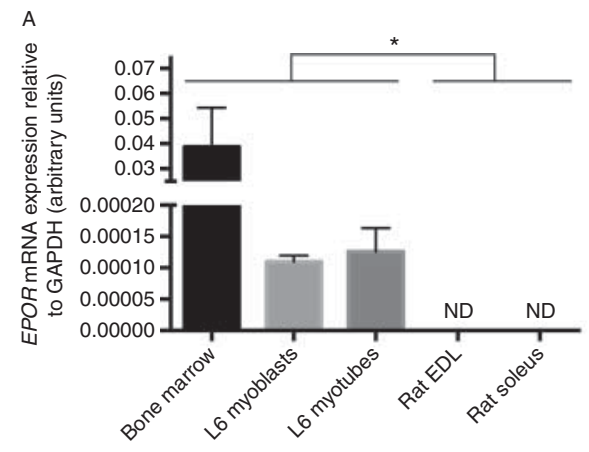

Figure 6

While EPOR is expressed in muscle cells, EPO does not stimulate glucose uptake or glycogen synthesis in cultured myotubes. (A) EPOR expression in bone marrow, muscle (soleus and EDL), myoblasts, and myotubes.

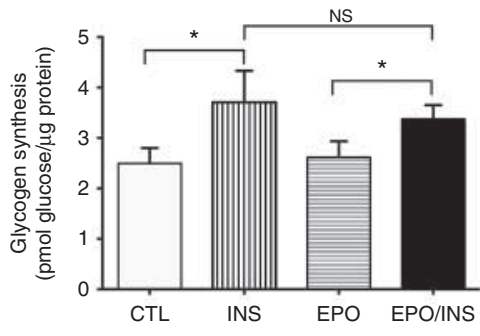

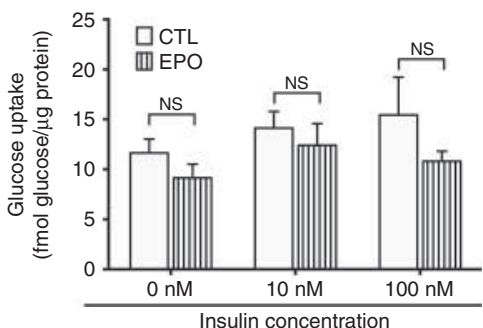

(B) Insulin-stimulated glycogen synthesis and (C) glucose uptake in L6 myotubes incubated with or without EPO. ${ }^{\star} P<0.05$ indicates a statistically significant difference between groups. NS, nonsignificant. http://joe.endocrinology-journals.org DOI: 10.1530/JOE-15-0010
(C) 2015 The authors Printed in Great Britain
Published by Bioscientifica Ltd 
A
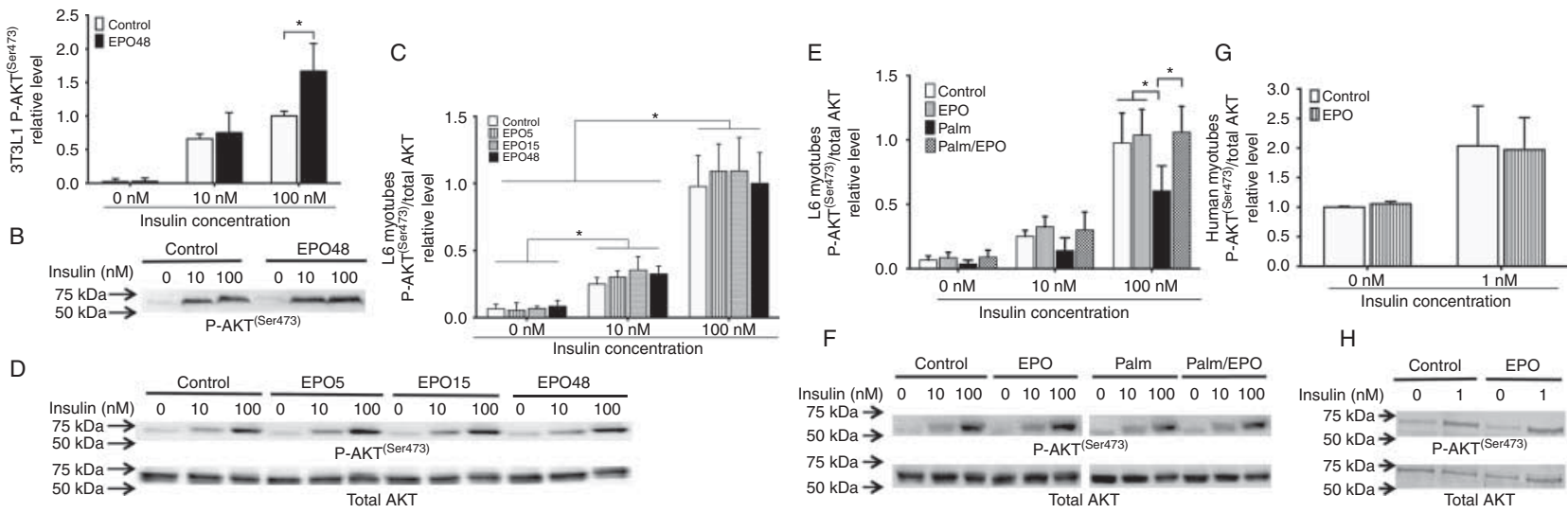

Figure 7

EPO does not activate AKT phosphorylation in cultured myotubes. (A) Quantifications and (B) representative western blot of P-AKT in response to insulin in 3T3L1 adipocytes under control or EPO-treated conditions for $48 \mathrm{~h}(n=3)$. (C) Quantifications and (D) representative western blot of P-AKT/total AKT in response to insulin in $\mathrm{L} 6$ myotubes incubated under control or EPO-treated conditions for $5 \mathrm{~min}, 15 \mathrm{~min}$, or $48 \mathrm{~h}(n=4)$. (E) Quantification and (F) representative western blot image of

P-AKT/total AKT in response to insulin in $L 6$ myotubes incubated with EPO, palmitate, or EPO/palmitate. (G) Quantifications and $(\mathrm{H})$ representative western blot image of P-AKT/total AKT in response to 0 or $1 \mathrm{nM}$ insulin in human primary myotubes incubated with or without EPO $(n=3) .{ }^{*} P<0.05$ between conditions as shown on figure. EPO5, EPO15, and EPO48: incubation with EPO for $5 \mathrm{~min}, 15 \mathrm{~min}$, or $48 \mathrm{~h}$ respectively. Palm, incubation with palmitate; Palm/EPO, incubation with palmitate and EPO.

blood glucose, and insulin levels as well as impaired glucose tolerance. It is noteworthy that while EPO treatment improved glucose control, it did not change body weight and retroperitoneal fat mass. This indicates that $\mathrm{EPO}$ ameliorates glucose metabolism independently from changes in body composition. Such effect can be explained via direct action on metabolic tissues, or indirectly through EPO anti-inflammatory effects (Li et al. 2006, Alnaeeli et al. 2014). Partial recovery of glucose tolerance was accompanied by recovery of baseline glucose levels in the HFD/EPO group while insulin levels were not yet affected. It is tempting to speculate that we observed early adaptations, and that longer EPO treatment or larger doses may be necessary to normalize insulin levels and to restore normal glucose metabolism (Katz et al. 2010). Our results also indicated that EPO treatment restored insulin-stimulated AKT activation in skeletal muscles in response to insulin in the HFD/EPO group in oxidative muscle (soleus); however, this did not lead to a statistically significant increase in P-AKT levels when compared with HFD due to increased baseline levels in this group. In contrast, EDL, a muscle characterized by glycolytic metabolism, did not show any response to EPO treatment. As EPOR mRNA was undetectable in soleus or $\mathrm{EDL}$, we conclude that any effect of EPO treatment on muscle AKT phosphorylation is indirect and results from lower systemic inflammation, reduced muscle oxidative stress, or increased HSP72 expression, all known to

positively influence insulin signaling. On the other hand, other metabolic tissues, such as liver (Meng et al. 2013) or adipose tissue (Mikolás et al. 2012, Wang et al. 2013), which have been shown to express EPOR and respond to EPO, can also participate in improved glucose control in our model.

Our results from cultured cells confirm that EPO can stimulate AKT activation in synergy with insulin in 3T3L1 adipocytes and demonstrate the lack of direct action of EPO on muscle cells. Indeed, we show that EPO did not affect AKT phosphorylation when rat L6 or human primary myotubes were incubated with EPO or coincubated with both EPO and insulin, indicating that EPO did not directly target AKT in differentiated skeletal muscle cells. In agreement with the lack of effect of EPO on AKT activation in myotubes, we also demonstrate that EPO did not increase glucose uptake or glycogen synthesis. However, we showed that EPO had the potential to rescue AKT phosphorylation when cells were subjected to palmitate treatment, a model used to mimic in vitro the deleterious effect of HFD on insulin signaling. While the underlying mechanism cannot be determined from our results, these indicate that EPO signaling in myotubes may provide some protection to the insulin signaling cascade. Expression of the EPOR gene has been long thought to be restricted to the erythroid lineage but has since been identified in C2C12 myoblasts (Ogilvie et al. 2000, Launay et al. 2010, Jia et al. 2012) and myotubes

Published by Bioscientifica Ltd 
(Joshi et al. 2011, Jia et al. 2012), as well as in rat (Rotter et al. 2008) and human skeletal muscle (Lundby et al. 2008, Rundqvist et al. 2009). Although chronic treatment with $\mathrm{EPO}$ in vivo resulted in increased AKT activation (Hojman et al. 2009) and enhanced mitochondrial oxidative phosphorylation (Plenge et al. 2012) in mouse and human skeletal muscle respectively, it is still unclear whether EPOR is functional and leads to a direct effect of EPO in muscle cells. Our investigation revealed expression of EPOR mRNA in rat L6 myotubes, although to a lesser extent than that in myoblasts, while soleus and EDL muscles displayed virtually no expression. Taken together, these results indicate that EPO does not directly modulate AKT activation in rat soleus muscle.

The mechanisms that contribute to the improvement of glucose tolerance and muscle insulin signaling in HFD/EPOtreated animals may also include decreased systemic inflammation, as indicated by reduced TNF $\alpha$ levels, the lower muscle oxidative stress, and enhanced expression of chaperone protein HSP72 observed in muscles from highfat-fed EPO-treated animals. TNF $\alpha$, which is produced by inflamed adipose tissue, is one of the pro-inflammatory cytokines involved in obesity-associated low grade inflammation (Shoelson et al. 2006). Its effect on insulin sensitivity and insulin signaling pathway activation in skeletal muscle is well documented (Plomgaard et al. 2005, Hotamisligil 2006). While other pro-inflammatory cytokines such as IL6 or IL1 $\beta$ (Li et al. 2008) could be involved, the reduced plasma TNF $\alpha$ levels observed in this study indicate that EPO improves insulin signaling and glucose tolerance through, at least in part, lowering of TNF $\alpha$ levels. In our study model, lower TNF $\alpha$ levels may be associated with reduced macrophage infiltration in adipose tissue as previously reported (Alnaeeli et al. 2014). It has been reported that EPO can attenuate plasma TNF $\alpha$ in hemodialyzed patients (RasicMilutinovic et al. 2008), and hamper TNF $\alpha$ production by 3T3L1 adipocytes (Pan et al. 2013). In mice fed a HFD, a 2-week EPO treatment has been reported to reduce inflammation and modulate PI3K/AKT signaling in liver (Meng et al. 2013). This indicates that EPO lowers HFD-associated inflammation and that additional tissues may play a role in improving glucose regulation in our model. In addition, EPO treatment of HFD animals decreased oxidative stress, as indicated by reduced levels of IsoFs, and also increased HSP72 expression in soleus muscle. The modulation of oxidative stress and the restoration of HSP72 could both mediate the protective effect of EPO against lipotoxicity. The expression of HSP72 is decreased in obesity (Chung et al. 2008, Gupte et al. 2009) or T2D (Bruce et al. 2003) and associated with insulin resistance.
Conversely, overexpression or restoration of this protein improves insulin sensitivity (Chung et al. 2008, Gupte et al. 2009). The mechanisms involved in the reduced expression of HSP72 in skeletal muscle in T2D are still unclear, but seem to be driven by a systemic inflammatory state (Chung et al. 2008).

In conclusion, we show that a 2-week EPO treatment in rats fed a HFD can improve glucose tolerance independently of body weight and retroperitoneal fat content. We observed incomplete restoration of insulinstimulated AKT activation in muscle in vivo; however, given the absence of EPOR expression in rat soleus and EDL muscles and the absence of AKT activation in myotubes exposed to EPO, we conclude that EPO does not target AKT directly in skeletal muscle. The observed reduced systemic TNF $\alpha$ level, and oxidative stress, as well as the restoration of HSP72 expression in soleus may act in synergy to provide protection against the diet-induced insulin resistance in metabolic tissues and contribute to the positive action of EPO on glucose homeostasis.

\section{Declaration of interest}

The authors declare that there is no conflict of interest that could be perceived as prejudicing the impartiality of the research reported.

\section{Funding}

Dr C C acknowledges support from 2013 Thompson Fellowship, University of Sydney. This study was partly funded by a Cumberland Research Grant (University of Sydney) and by the Novo Nordisk Foundation. The Novo Nordisk Foundation Centre for Basic Metabolic Research is an independent Research Centre at the University of Copenhagen partially funded by an unrestricted donation from the Novo Nordisk Foundation (www.metabol. ku.dk). H A was awarded an Albert Renold Travel Fellowship (European Foundation for the Study of Diabetes).

\section{Author contribution statement}

$C C, R B$, and D S designed the studies. C C, H A, E M, M M, A N M, and P R conducted the experiments. C C, R B, H A, J M, C B, T A M, and S T analyzed the results. $C C, R B$, and D S wrote the manuscript and all authors approved the final version of the manuscript.

\section{Acknowledgements}

The authors would like to thank Dr Kieron Rooney for his valuable comments during the course of the study. They also acknowledge the support provided by Dr Remi Thomasson.

\section{References}

Al-Khalili L, Chibalin AV, Kannisto K, Zhang BB, Permert J, Holman GD, Ehrenborg E, Ding VD, Zierath JR \& Krook A 2003 Insulin action in

Published by Bioscientifica Ltd 
cultured human skeletal muscle cells during differentiation: assessment of cell surface GLUT4 and GLUT1 content. Cellular and Molecular Life Sciences 60 991-998. (doi:10.1007/s00018-003-3001-3)

Alnaeeli M, Raaka BM, Gavrilova O, Teng R, Chanturiya T \& Noguchi CT 2014 Erythropoietin signaling: a novel regulator of white adipose tissue inflammation during diet-induced obesity. Diabetes 63 2415-2431. (doi:10.2337/db13-0883)

Barrès R, Osler ME, Yan J, Rune A, Fritz T, Caidahl K, Krook A \& Zierath JR 2009 Non-CpG methylation of the $P G C-1 \alpha$ promoter through DNMT3B controls mitochondrial density. Cell Metabolism 10 189-198. (doi:10.1016/j.cmet.2009.07.011)

Barrès R, Yan J, Egan B, Treebak JT, Rasmussen M, Fritz T, Caidahl K, Krook A, O'Gorman DJ \& Zierath JR 2012 Acute exercise remodels promoter methylation in human skeletal muscle. Cell Metabolism 15 405-411. (doi:10.1016/j.cmet.2012.01.001)

Barro M, Carnac G, Flavier S, Mercier J, Vassetzky Y \& Laoudj-Chenivesse D 2010 Myoblasts from affected and non-affected FSHD muscles exhibit morphological differentiation defects. Journal of Cellular and Molecular Medicine 14 275-289. (doi:10.1111/j.1582-4934.2008.00368.x)

Borissova AM, Djambazova A, Todorov K, Dakovska L, Tankova T \& Kirilov G 1993 Effect of erythropoietin on the metabolic state and peripheral insulin sensitivity in diabetic patients on haemodialysis. Nephrology, Dialysis, Transplantation 893.

Bruce CR, Carey AL, Hawley JA \& Febbraio MA 2003 Intramuscular heat shock protein 72 and heme oxygenase-1 mRNA are reduced in patients with type 2 diabetes: evidence that insulin resistance is associated with a disturbed antioxidant defense mechanism. Diabetes 52 2338-2345. (doi:10.2337/diabetes.52.9.2338)

Christensen B, Lundby C, Jessen N, Nielsen TS, Vestergaard PF, Møller N, Pilegaard H, Pedersen SB, Kopchick JJ \& Jørgensen JO 2012 Evaluation of functional erythropoietin receptor status in skeletal muscle in vivo: acute and prolonged studies in healthy human subjects. PLOS ONE $\mathbf{7}$ e31857. (doi:10.1371/journal.pone.0031857)

Chung J, Nguyen AK, Henstridge DC, Holmes AG, Chan MH, Mesa JL, Lancaster GI, Southgate RJ, Bruce CR, Duffy SJ et al. 2008 HSP72 protects against obesity-induced insulin resistance. PNAS $\mathbf{1 0 5}$ 1739-1744. (doi:10.1073/pnas.0705799105)

Gupte AA, Bomhoff GL, Swerdlow RH \& Geiger PC 2009 Heat treatment improves glucose tolerance and prevents skeletal muscle insulin resistance in rats fed a high-fat diet. Diabetes 58 567-578. (doi:10.2337/ db08-1070)

Henriksen EJ, Diamond-Stanic MK \& Marchionne EM 2011 Oxidative stress and the etiology of insulin resistance and type 2 diabetes. Free Radical Biology \& Medicine 51 993-999. (doi:10.1016/j.freeradbiomed.2010.12.005)

Hojman P, Brolin C, Gissel H, Brandt C, Zerahn B, Pedersen BK \& Gehl J 2009 Erythropoietin over-expression protects against diet-induced obesity in mice through increased fat oxidation in muscles. PLoS ONE 4 e5894. (doi:10.1371/journal.pone.0005894)

Hotamisligil GS 2006 Inflammation and metabolic disorders. Nature $\mathbf{4 4 4}$ 860-867. (doi:10.1038/nature05485)

Jia Y, Suzuki N, Yamamoto M, Gassmann M \& Noguchi CT 2012 Endogenous erythropoietin signaling facilitates skeletal muscle repair and recovery following pharmacologically induced damage. FASEB Journal 26 2847-2858. (doi:10.1096/fj.11-196618)

Joshi D, Patel H, Baker DM, Shiwen X, Abraham DJ \& Tsui JC 2011 Development of an in vitro model of myotube ischemia. Laboratory Investigation 91 1241-1252. (doi:10.1038/labinvest.2011.79)

Katz O, Stuible M, Golishevski N, Lifshitz L, Tremblay ML, Gassmann M, Mittelman M \& Neumann D 2010 Erythropoietin treatment leads to reduced blood glucose levels and body mass: insights from murine models. Journal of Endocrinology 205 87-95. (doi:10.1677/JOE-09-0425)

Khedr E, El-Sharkawy M, Abdulwahab S, Eldin EN, Ali M, Youssif A \& Ahmed B 2009 Effect of recombinant human erythropoietin on insulin resistance in hemodialysis patients. Hemodialysis International $\mathbf{1 3}$ 340-346. (doi:10.1111/j.1542-4758.2009.00367.x)
Launay T, Hagström L, Lottin-Divoux S, Marchant D, Quidu P, Favret F, Duvallet A, Darribere T, Richalet J-P \& Beaudry M 2010 Blunting effect of hypoxia on the proliferation and differentiation of human primary and rat L6 myoblasts is not counteracted by Epo. Cell Proliferation $\mathbf{4 3}$ 1-8. (doi:10.1111/j.1365-2184.2009.00648.x)

Li Y, Takemura G, Okada H, Miyata S, Maruyama R, Li L, Higuchi M, Minatoguchi S, Fujiwara T \& Fujiwara H 2006 Reduction of inflammatory cytokine expression and oxidative damage by erythropoietin in chronic heart failure. Cardiovascular Research 71 684-694. (doi:10.1016/j.cardiores.2006.06.003)

Li H, Lelliott C, Håkansson P, Ploj K, Tuneld A, Verolin-Johansson M, Benthem L, Carlsson B, Storlien L \& Michaëlsson E 2008 Intestinal, adipose, and liver inflammation in diet-induced obese mice. Metabolism: Clinical and Experimental 57 1704-1710. (doi:10.1016/j.metabol.2008.07.029)

Lundby C, Hellsten Y, Jensen MB, Munch AS \& Pilegaard H 2008 Erythropoietin receptor in human skeletal muscle and the effects of acute and long-term injections with recombinant human erythropoietin on the skeletal muscle. Journal of Applied Physiology 104 1154-1160. (doi:10.1152/japplphysiol.01211.2007)

Maiese K, Li F \& Chong ZZ 2005 New avenues of exploration for erythropoietin. Journal of the American Medical Association 293 90-95. (doi:10.1001/jama.293.1.90)

Mak RH 1996 Correction of anemia by erythropoietin reverses insulin resistance and hyperinsulinemia in uremia. American Journal of Physiology 270 F839-F844.

Marchionne EM, Diamond-Stanic MK, Prasannarong M \& Henriksen EJ 2012 Chronic renin inhibition with aliskiren improves glucose tolerance, insulin sensitivity, and skeletal muscle glucose transport activity in obese Zucker rats. American Journal of Physiology. Regulatory, Integrative and Comparative Physiology 302 R137-R142. (doi:10.1152/ ajpregu.00448.2011)

Mas E, Barden AE, Corcoran TB, Phillips M, Roberts LJ \& Mori TA 2011 Effects of spinal or general anesthesia on $\mathrm{F}_{2}$-isoprostanes and isofurans during ischemia/reperfusion of the leg in patients undergoing knee replacement surgery. Free Radical Biology \& Medicine 50 1171-1176. (doi:10.1016/j.freeradbiomed.2011.01.021)

Meng R, Zhu D, Bi Y, Yang D \& Wang Y 2013 Erythropoietin inhibits gluconeogenesis and inflammation in the liver and improves glucose intolerance in high-fat diet-fed mice. PLoS ONE 8 e53557. (doi:10.1371/ journal.pone.0053557)

Mikolás E, Cseh J, Pap M, Szijárto IA, Balogh A, Laczy B, Bekő V, Fisi V, Molnár GA, Mérei A et al. 2012 Effects of erythropoietin on glucose metabolism. Hormone and Metabolic Research 44 279-285. (doi:10.1055/ s-0032-1301901)

Montuschi P, Barnes PJ \& Roberts LJ 2004 Isoprostanes: markers and mediators of oxidative stress. FASEB Journal 18 1791-1800. (doi:10.1096/fj.04-2330rev)

Morrow JD, Hill KE, Burk RF, Nammour TM, Badr KF \& Roberts LJ II 1990 A series of prostaglandin $\mathrm{F}_{2}$-like compounds are produced in vivo in humans by a non-cyclooxygenase, free radical-catalyzed mechanism. PNAS 87 9383-9387. (doi:10.1073/pnas.87.23.9383)

Ogilvie M, Yu X, Nicolas-Metral V, Pulido SM, Liu C, Ruegg UT \& Noguchi CT 2000 Erythropoietin stimulates proliferation and interferes with differentiation of myoblasts. Journal of Biological Chemistry 275 39754-39761. (doi:10.1074/jbc.M004999200)

Pan Y, Shu JL, Gu HF, Zhou DC, Liu XL, Qiao QY, Fu SK, Gao FH \& Jin HM 2013 Erythropoietin improves insulin resistance via the regulation of its receptor-mediated signaling pathways in 3T3L1 adipocytes. Molecular and Cellular Endocrinology 367 1-42. (doi:10.1016/j.mce.2012.12.027)

Plenge U, Belhage B, Guadalupe-Grau A, Andersen PR, Lundby C, Dela F, Stride N, Pott FC, Helge JW \& Boushel R 2012 Erythropoietin treatment enhances muscle mitochondrial capacity in humans. Frontiers in Physiology 3 1-5. (doi:10.3389/fphys.2012.00050)

Plomgaard P, Bouzakri K, Krogh-Madsen R, Mittendorfer B, Zierath JR \& Pedersen BK 2005 Tumor necrosis factor- $\alpha$ induces skeletal muscle insulin resistance in healthy human subjects via inhibition of Akt 
substrate 160 phosphorylation. Diabetes 54 2939-2945. (doi:10.2337/ diabetes.54.10.2939)

Rasic-Milutinovic Z, Perunicic-Pekovic G, Cavala A, Gluvic Z, Bokan L \& Stankovic S 2008 The effect of recombinant human erythropoietin treatment on insulin resistance and inflammatory markers in nondiabetic patients on maintenance hemodialysis. Hippokratia 12 157-161.

Rotter R, Menshykova M, Winkler T, Matziolis G, Stratos I, Schoen M, Bittorf T, Mittlmeier T \& Vollmar B 2008 Erythropoietin improves functional and histological recovery of traumatized skeletal muscle tissue. Journal of Orthopaedic Research 26 1618-1626. (doi:10.1002/ jor.20692)

Rundqvist H, Rullman E, Sundberg CJ, Fischer H, Eisleitner K, Ståhlberg M, Sundblad P, Jansson E \& Gustafsson T 2009 Activation of the erythropoietin receptor in human skeletal muscle. European Journal of Endocrinology 161 427-434. (doi:10.1530/EJE-09-0342)

Shoelson SE, Lee J \& Goldfine AB 2006 Inflammation and insulin resistance. Journal of Clinical Investigation 116 1793-1801. (doi:10.1172/ JCI29069)
Spaia S, Pangalos M, Askepidis N, Pazarloglou M, Mavropoulou E, Theodoridis S, Dimitrakopoulos K, Milionis A \& Vayonas G 2000 Effect of short-term rHuEPO treatment on insulin resistance in haemodialysis patients. Nephron 84 320-325. (doi:10.1159/000045606)

Tuzcu A, Bahceci M, Yilmaz E, Bahceci S \& Tuzcu S 2004 The comparison of insulin sensitivity in non-diabetic hemodialysis patients treated with and without recombinant human erythropoietin. Hormone and Metabolic Research 36 716-720. (doi:10.1055/s-2004826021)

Wang L, Teng R, Di L, Rogers H, Wu H, Kopp JB \& Noguchi CT 2013 PPAR $\alpha$ and Sirt1 mediate erythropoietin action in increasing metabolic activity and browning of white adipocytes to protect against obesity and metabolic disorders. Diabetes 62 4122-4131. (doi:10.2337/ db13-0518)

Wang L, Di L \& Noguchi CT 2014 Erythropoietin, a novel versatile player regulating energy metabolism beyond the erythroid system. International Journal of Biological Sciences 10 921-939. (doi:10.7150/ ijbs.9518)

Received in final form 6 March 2015

Accepted 12 March 2015

Accepted Preprint published online 12 March 2015
Published by Bioscientifica Ltd 